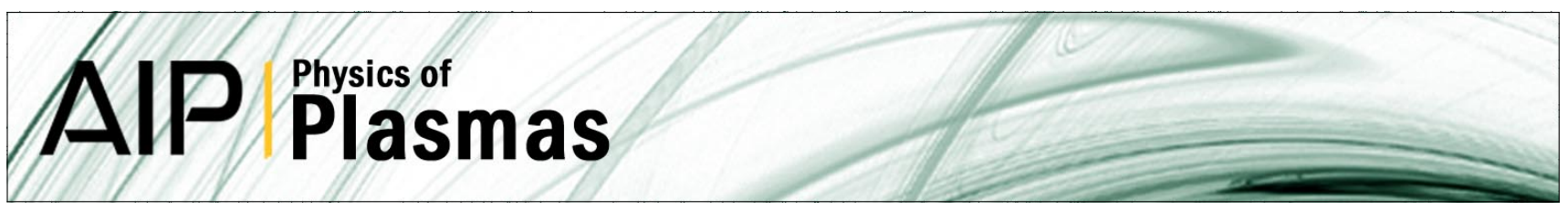

\title{
Acceleration of laser-driven ion bunch from double-layer thin foils
}

X. Wang, W. Yu, E. Liang, and M. Y. Yu

Citation: Phys. Plasmas 19, 053110 (2012); doi: 10.1063/1.4714613

View online: http://dx.doi.org/10.1063/1.4714613

View Table of Contents: http://pop.aip.org/resource/1/PHPAEN/v19/i5

Published by the American Institute of Physics.

\section{Related Articles}

Ion impact distribution over plasma exposed nanocone arrays

Phys. Plasmas 20, 033501 (2013)

Enhanced transportation of energetic electrons in dual-frequency atmospheric microplasmas

Phys. Plasmas 20, 023506 (2013)

Suppressing longitudinal double-layer oscillations by using elliptically polarized laser pulses in the hole-boring radiation pressure acceleration regime

Phys. Plasmas 20, 023102 (2013)

Two-dimensional quasi-double-layers in two-electron-temperature, current-free plasmas

Phys. Plasmas 20, 023502 (2013)

Evidence of current free double layer in high density helicon discharge

Phys. Plasmas 20, 013510 (2013)

\section{Additional information on Phys. Plasmas}

Journal Homepage: http://pop.aip.org/

Journal Information: http://pop.aip.org/about/about_the_journal

Top downloads: http://pop.aip.org/features/most_downloaded

Information for Authors: http://pop.aip.org/authors

\section{ADVERTISEMENT}

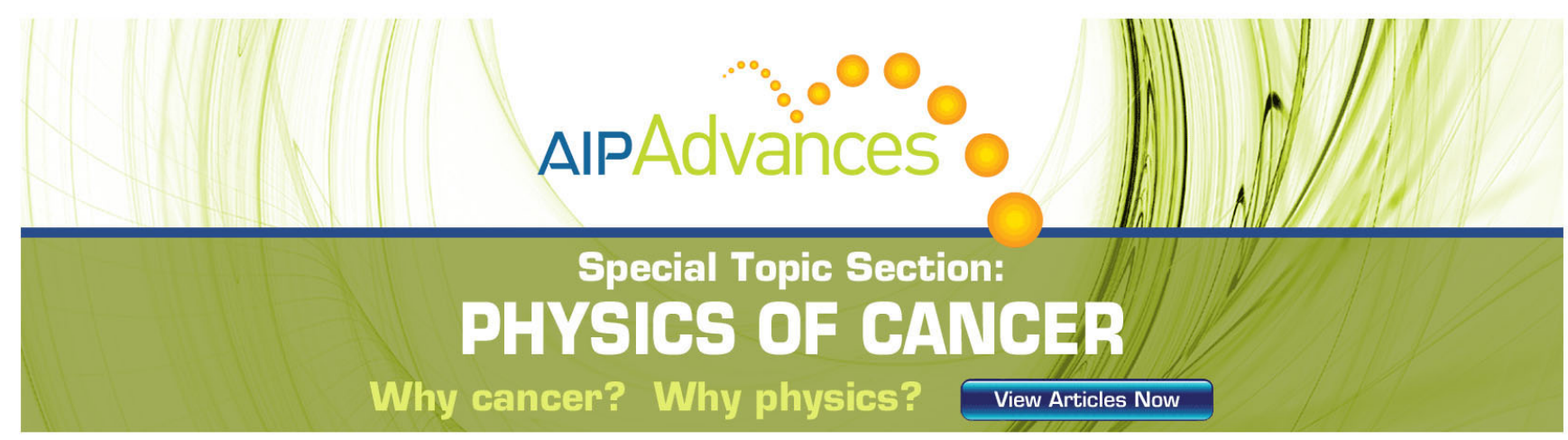




\title{
Acceleration of laser-driven ion bunch from double-layer thin foils
}

\author{
X. Wang (王拿金), ${ }^{1}$ W. Yu (余玮), ${ }^{2}$ E. Liang, ${ }^{1}$ and M. Y. Yu (郁明阳) ${ }^{3}$ \\ ${ }^{1}$ Rice University, Houston, Texas 77005-1892, USA \\ ${ }^{2}$ Shanghai Institute of Optics and Fine Mechanics, Chinese Academy of Sciences, Shanghai 201800, China \\ ${ }^{3}$ Institute for Fusion Theory and Simulation, Zhejiang University, Hangzhou 310027, China
}

(Received 9 January 2012; accepted 10 April 2012; published online 23 May 2012)

Generation of monoenergetic ion bunch from a double-layer thin-foil target irradiated by an intense linearly polarized laser pulse is investigated using two-dimensional particle-in-cell simulation. The protons in the front low-density hydrogen target layer accelerated by the space-charge field of the laser-driven hot electrons can penetrate through the high- $Z$ high-mass and high-density ion layer, resulting in an energetic proton bunch. A part of the latter is further accelerated by the space-charge field of the hot electrons in the vacuum behind the high- $Z$ ion layer. With this scheme, quasimonoenergetic proton bunches can be produced using presently available laser pulses of moderate contrast and duration. (C) 2012 American Institute of Physics. [http://dx.doi.org/10.1063/1.4714613]

\section{INTRODUCTION}

In recent years, the availability of ultrashort ultraintense (USUI) laser pulse makes possible the development of compact laser-driven ion accelerators. ${ }^{1-3}$ Energetic ions have many important applications, including proton therapy, ${ }^{4}$ diagnostics for laser-plasma interaction, ${ }^{5}$ and fast ignition in inertial-confinement fusion. ${ }^{6}$ USUI laser interaction with plasma has also been proposed for laboratory investigation of high energy density physics, especially that of some astrophysical phenomena. For most of these applications, energetic ions of sufficiently high energy and brightness are required.

Several acceleration mechanisms for generating high quality ion bunches have been proposed, including target normal sheath acceleration (TNSA), ${ }^{7-10}$ radiation pressure acceleration (RPA), ${ }^{11-18}$ Coulomb explosion, ${ }^{19}$ and breakout afterburner acceleration, ${ }^{20}$ as well as combinations of one or more mechanisms. TNSA occurs when an USUI laser pulse irradiates a solid foil and the intense sheath field of the laser-expelled hot electrons in the target-backside vacuum can pull out the ions in the back surface of the target and accelerate them to the $\mathrm{MeV}$ level. ${ }^{21-28}$ With the rapid recent development of laser technology, such as that of the plasma mirror, $^{29}$ the RPA scheme has drawn increased attention since it can generate ion bunches with high density and energy. But instability suppression and bunch confinement remain unsolved, especially if linearly polarized (LP) lasers are used.

In this paper, we consider a new ion acceleration mechanism that combines TNSA and RPA to generate quasimonoenergetic ion bunches in LP laser interaction with a thin double-layer target ${ }^{30,31}$ The latter consists of a lowdensity low- $Z$ light-ion layer with a high-density high- $Z$ heavy-ion layer at its rear, such that TNSA and RPA can take place simultaneously. It is shown that laser-energy absorption by such a composite target is much improved from that by a simple target. ${ }^{34}$ More importantly, the stringent conditions on the laser pulse duration, polarization, as well as contrast can be much relaxed.

\section{PARTICLE-IN-CELL (PIC) SIMULATION PARAMETERS}

For the investigation, we shall use 2D3V (two dimensional in space and three dimensional in velocity) PIC simulation. ${ }^{32,33}$ A short LP laser pulse of wavelength $\lambda_{0}=1 \mu \mathrm{m}$ is incident normally on the double-layer target along the $z$ direction from the left vacuum. The laser intensity, modeling the Texas Petawatt Laser, ${ }^{35}$ is $I=I_{0}=3 \times 10^{20} \exp$ $\left(-4 r^{2} / d^{2}\right) \mathrm{Wcm}^{-2}$ for $15 T_{0}<t<45 T_{0}$, where $T_{0}=3.3 \mathrm{fs}$ is the wave period, $d=10 \lambda_{0}$ is the spot size, and $I=$ $I_{0} \sin ^{2}(\pi t / 2 \tau)$ for $0<t<15 T_{0}$ and $45 T_{0}<t<60 T_{0}$, here $\tau=15 T_{0}$ is the laser ascend or descend duration. The laser parameter and pulse duration are therefore $a_{L}=15$ and $45 T_{0}$, respectively. The low- $Z$ plasma on the front side of the target is hydrogen $(Z=1)$ with electron density $n_{e}=4 n_{c}$, which is much lower than that of the backside high- $Z$ highdensity large-mass ion layer. The latter is aluminum (Al, with $Z=13$ ) with electron density $n_{e}=650 n_{c}$ (i.e., the $\mathrm{Al}$ ion density is $n_{i}=50 n_{c}$ ). Initially, both the $\mathrm{H}$ and Al layers are of thickness $0.1 \lambda_{0}$, and are located in $4.9 \leq z / \lambda_{0} \leq 5.0$ and $5.0<z / \lambda_{0} \leq 5.1$, respectively. The $\mathrm{H}$ plasma critical density is $n_{c}=1.1 \times 10^{21} \mathrm{~cm}^{-3}$. Accordingly, the nonrelativistic skin depths of the $\mathrm{H}$ and $\mathrm{Al}$ plasmas are $0.08 \lambda_{0}$ and $0.006 \lambda_{0}$, respectively.

The simulation box is $30 \lambda_{0} \times 19.2 \lambda_{0}$, the spatial mesh contains $3000 \times 1920$ cells, and each cell contains 625 each of ions and electrons. The electron-ion mass ratios are $1 / 1836$ for $\mathrm{H}$ and $1 / 49572$ for $\mathrm{Al}$. The initial temperature of all the plasma particles is assumed to be $T_{e}=T_{i}=1 \mathrm{keV}$, although the electrons can be rapidly heated during the initial stages of the interaction. The simulation time step is $0.006 T_{0}$. Absorbing boundary conditions are used for both the $y$ and $z$ boundaries of the simulation box.

\section{SIMULATION RESULTS AND DISCUSSIONS}

The dominant interaction forces here are the relativistic ponderomotive force action on the electrons and the chargeseparation forces acting on the ions and electrons. Since our 
PIC code does not include close (such as binary) collisions among the charged particles, we first estimate its validity for the present problem. From Wesson, ${ }^{36}$ one can obtain for the electron and ion mean free paths $3270 \frac{T_{e}^{3 / 2}\left(1022 T_{e}+T_{e}^{2}\right)^{1 / 2}}{n_{i} Z_{i}^{2}\left(511+T_{e}\right) \ln \Lambda_{e}} \mu \mathrm{m}$ and $1.98 \times 10^{5} \frac{A^{1 / 2} T_{i}^{3 / 2}\left(1022 a T_{i}+T_{i}^{2}\right)^{1 / 2}}{n_{i} Z_{i}^{4}\left(511 a+T_{e}\right) \ln \Lambda_{i}} \mu \mathrm{m}$, respectively, where $T_{e}, T_{i}$, $n_{i}, Z_{i}$, and $A$ are the electron temperature in $\mathrm{keV}$, ion temperature in $\mathrm{keV}$, ion density, ion charge number, and atomic number, respectively, and $a=m_{i} / m_{e}=1836$. The values of the Coulomb logarithms $\ln \Lambda_{e}$ and $\ln \Lambda_{i}$ are usually around 10. Thus, the Coulomb-collision cross section falls off rapidly for high-energy particles, especially the laser accelerated electrons. One finds that even a $1.4 \mathrm{keV}$ electron from the $\mathrm{H}$ target plasma can easily penetrate $0.1 \mu \mathrm{m}$ of the $\mathrm{Al}$ plasma, but for a proton, the energy required would be $35 \mathrm{keV}$. Accordingly, our PIC simulation, in which close (binary) collisions are not included, should be applicable.

The blue solid and green dotted curves in Fig. 1(a) show the spatial distribution of the charge densities (normalized by their initial values) of the $\mathrm{Al}$ and $\mathrm{H}$ plasma at $t=6 T_{0}$, respectively, together with that of the electrostatic chargeseparation field $E_{z}$. We see that most of the H-layer electrons (mainly represented by the negative part of the H-plasma charge density, see also the proton density profile in Fig. 2(a)) have already been driven into the high-density $\mathrm{Al}$ layer as
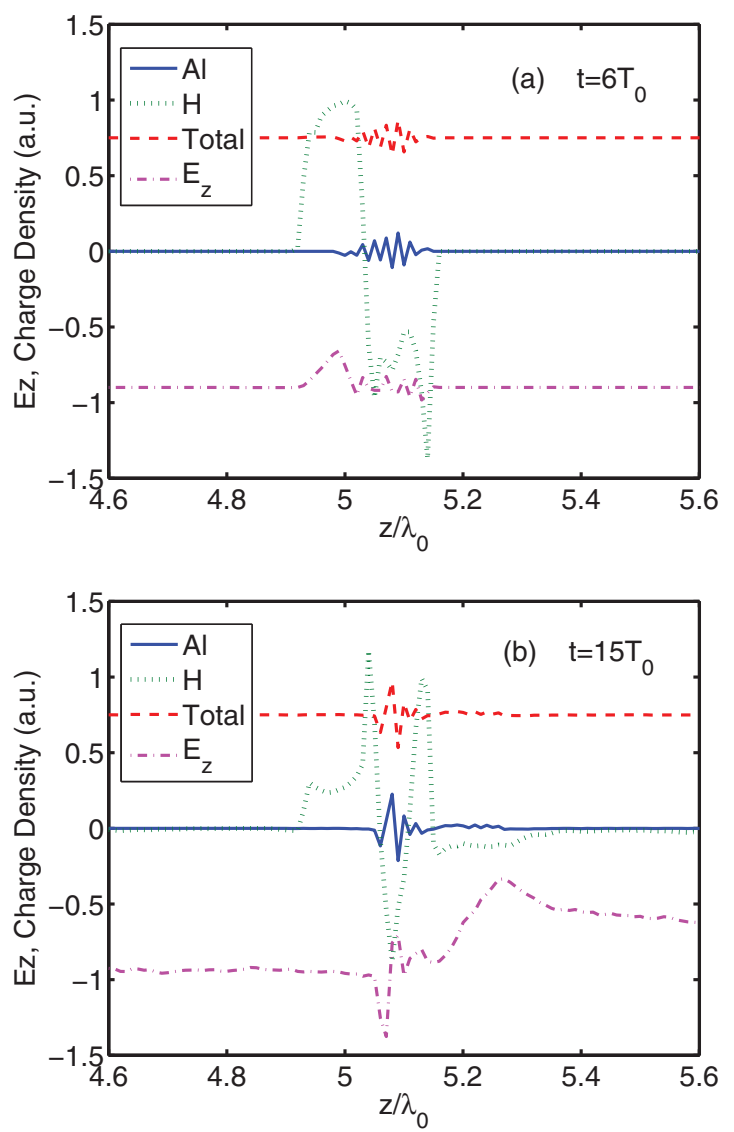

FIG. 1. Net charge-density distributions (normalized by their initial values) along the laser propagation axis $(y=0)$ at (a) $t=6 T_{0}$ and (b) $t=15 T_{0}$. The blue solid and green dotted curves show the charge densities of the $\mathrm{Al}$ and $\mathrm{H}$ plasmas, respectively, the red dashed and purple dot-dashed curves show the normalized total charge and $E_{z}$, respectively. For clarity, the latter are displaced by +0.75 and -0.9 , respectively.
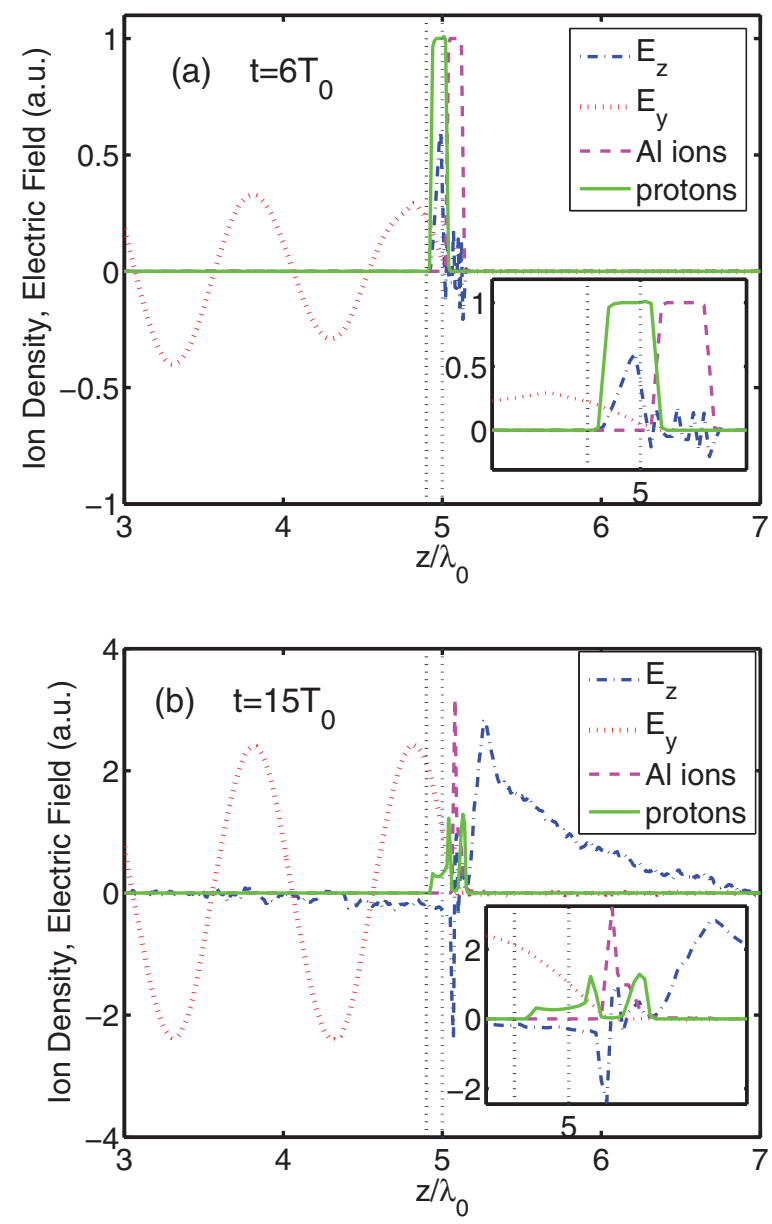

FIG. 2. $E_{y}$ (red dotted curve), $E_{z}$ (blue dot-dashed curve), proton density (green solid curve), and $\mathrm{Al}$ ion density (purple dashed curve) on $y=0$ at (a) $t=6 T_{0}$ and (b) $t=15 T_{0}$. Here, $E_{y}$ is normalized by the initial laser amplitude. The vertical dashed lines mark the front boundaries of the $\mathrm{H}$ and $\mathrm{Al}$ layers. For clarity, the insets show the magnified center regions of the figures.

well as the backside vacuum. These high-energy electrons can be attributed to ponderomotive acceleration by $\boldsymbol{v} \times \boldsymbol{B}$ force, relativistic effects, stochastic heating, vacuum heating, etc. The protons are then accelerated forward by TNSA. Although the laser light can easily penetrate the H-layer, it can only slightly tunnel through the Al layer (now with excess electrons), which is instead compressed and pushed forward by RPA. One can also observe charge density oscillations of the Al plasma, which can be attributed to acoustic motion excited by the passing energetic H-plasma electrons. As time progresses, more and more protons are accelerated into the $\mathrm{Al}$ layer and beyond. Eventually, the higher intensity part of the laser pulse arrives at the interface, Fig. 1(b) is for $t=15 T_{0}$. We can see that the Al layer has been strongly compressed and further pushed forward by RPA. Moreover, it splits the proton bunch that is expanding and moving, passing it into two groups (see also the density profiles of the protons and $\mathrm{Al}$ ions in Fig. 2(b)), as if the compressed $\mathrm{Al}$ plasma layer is trapped in a proton cavity. In the meantime, the now enhanced space-charge field in the backside vacuum continues to accelerate the protons, but now only that of the leading group since the trailing group is shielded by the Al layer. This scenario is consistent with the distributions of the field 

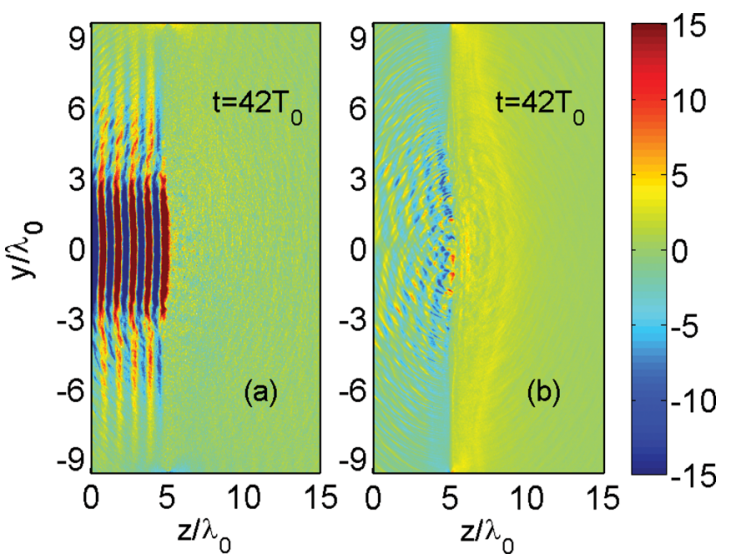

FIG. 3. 2D distributions of (a) the laser field $E_{y}$ and (b) the chargeseparation field $E_{z}$ at $t=42 T_{0}$.

quantities given in Fig. 2. In Fig. 2(a) for $t=6 T_{0}$, the red dotted curve represents the laser electric field $E_{y}$, normalized by its peak value. We can see that the laser light has penetrated into the $\mathrm{H}$ layer, whose thickness is comparable to the skin depth. Some light at the tip of the laser pulse has also tunneled through the Al layer, whose thickness is much more than the skin depth. The blue dotted-dashed curve shows the electrostatic field $E_{z}$, and the green solid and purple dashed curves show the $\mathrm{H}$ and $\mathrm{Al}$ ion densities, respectively. As mentioned, the laser expelled $\mathrm{H}$-layer electrons entering into the $\mathrm{Al}$ layer and the vacuum behind the target create chargeseparation electrostatic fields in the $\mathrm{H}$ and $\mathrm{Al}$ plasmas, as well as in the vacuum behind the target, so that the affected protons are driven forward by TNSA. Fig. 2(b) for $t=15 T_{0}$ shows that the Al plasma layer is indeed compressed by RPA, in fact to more than three times the original density. The main part of the laser cannot penetrate the $\mathrm{Al}$ layer and is absorbed as well as reflected. As mentioned, the strongly compressed Al plasma effectively split the protons into two groups. The leading group continues to propagate forward by TNSA as a nearly monoenergetic bunch. The trailing group is driven backwards in the $-z$ direction by the space-charge field of the electrons that have expanded into the left vacuum region.

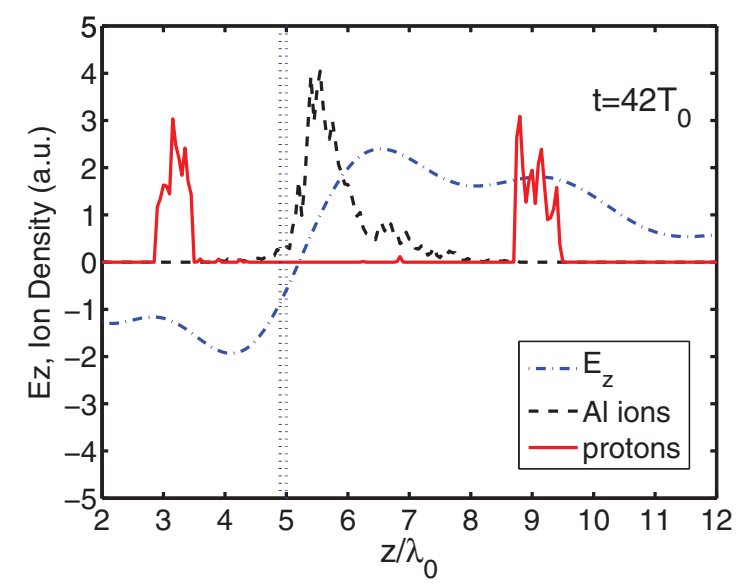

FIG. 4. The electrostatic field $E_{z}$ (blue dotted-dashed curve), and the $\mathrm{Al}$ ion (black dashed curve) and proton (red solid curve) densities on $y=0$ at $t=42 T_{0}$.
Fig. 3 for $E_{y}$ and $E_{z}$ at $t=42 T_{0}$ shows that, as the laser pulse compresses and accelerates the $\mathrm{Al}$ plasma by RPA, it is also absorbed (and reflected). The electrons of the highly compressed $\mathrm{Al}$ layer are resonantly heated and expand out of the layer but the much heavier $\mathrm{Al}$ ions respond much more slowly and remain in place. As a result, the space charge fields on both sides of the Al layer, as well as in the transverse directions, are widened and enhanced, as can be seen in Fig. 3(b). We can see in Fig. 4 that the distributions of the electrostatic field, $E_{z}$, and the proton and $\mathrm{Al}$ ion densities at $t=42 T_{0}$ are consistent with the discussed scenario.
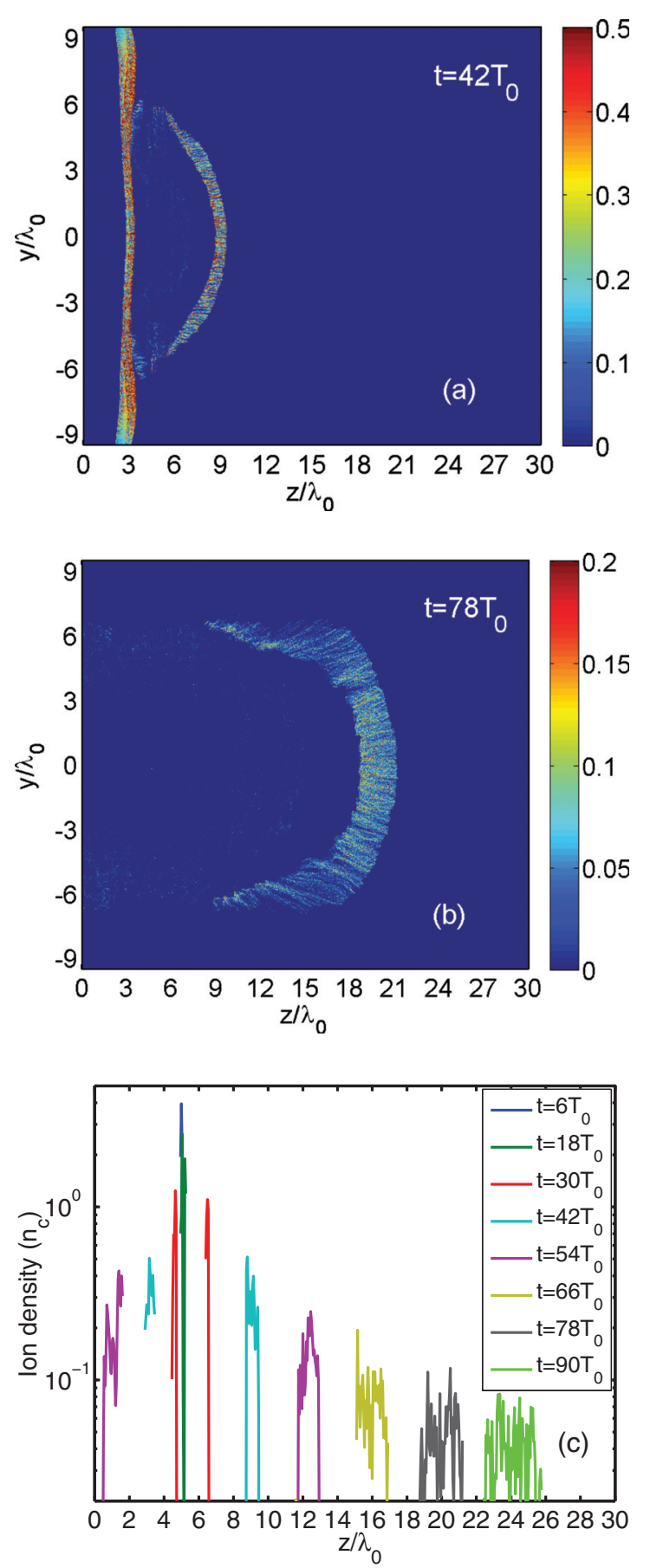

FIG. 5. 2D Proton density distribution at (a) $t=42 T_{0}$ and (b) $t=78 T_{0}$. (c) Proton density on $y=0$ at $t=6 T_{0}, 18 T_{0}, 30 T_{0}, 42 T_{0}, 54 T_{0}, 66 T_{0}, 78 T_{0}$, and $90 T_{0}$, respectively. The initial proton density is $n_{p}=4 n_{c}$. 
Thus, as the rest of the laser pulse interacts with the compressed Al plasma, the latter is further pushed forward by RPA and heated, and the quasistatic charge-separation field in the right vacuum continues to accelerate the leading proton bunch forward. We see that the present scheme efficiently converts laser energy into the electrostatic energy for accelerating the protons. Since the Al target prevents transmission of the laser pulse through the target, the electrostatic accelerating field and thus the proton bunch remain in the forward direction. The transverse motion of the protons is greatly reduced and Rayleigh-Taylor-like instabilities ${ }^{37}$ suppressed. Figs. 5(a) and 5(b) show the 2D proton density distributions at $t=42 T_{0}$ and $t=78 T_{0}$, respectively. Fig. 5(c) gives the proton densities on the axis $(y=0)$ at $12 T_{0}$ intervals. The initial proton density is $n_{p}=4 n_{c}$. We see that the average proton density is close to $0.05 n_{c}$ at $t=90 T_{0}$.

Figs. 6(a) and 6(b) shows the proton spectrum and average energy of the forward moving proton bunch at $t=6 T_{0}$, $18 T_{0}, 30 T_{0}, 42 T_{0}, 54 T_{0}, 66 T_{0}, 78 T_{0}$ and $90 T_{0}$. We see that, at $t=90 T_{0}$, the average proton energy is $\sim 55 \mathrm{MeV}$ (blue solid curve), and the most energetic (upper 40\%) of the forward moving protons have an average energy $\sim 65 \mathrm{MeV}$ (green dashed curve). The corresponding energy density is $5 \times 10^{8}$ $\mathrm{J} / \mathrm{cm}^{3}$, which should be suitable for many applications.

The proton energy spectrum can be improved by tailoring the relative sizes of the target layers. One way is to reduce the lateral extent of the hydrogen layer. Figure 7 is
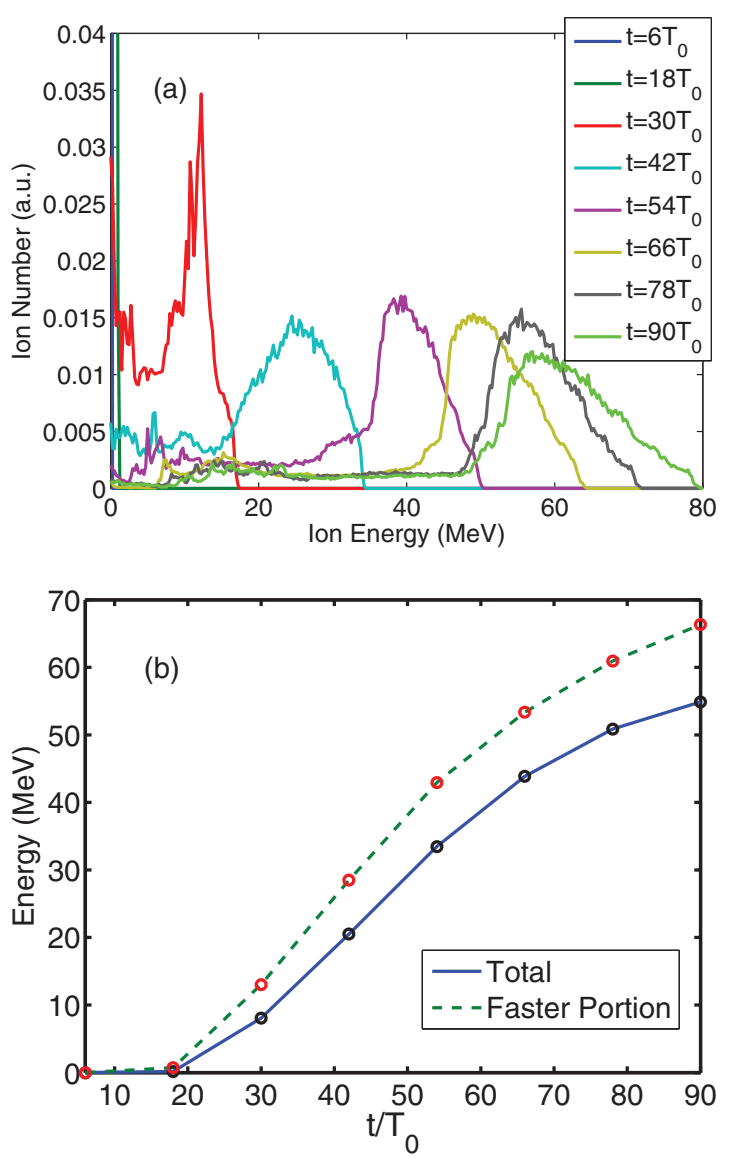

FIG. 6. (a) Evolution of the energy spectrum of the proton bunch and (b) the average energy of the forward propagating protons (blue solid curve) and average energy of the faster (upper 40\%) protons (green dashed curve).

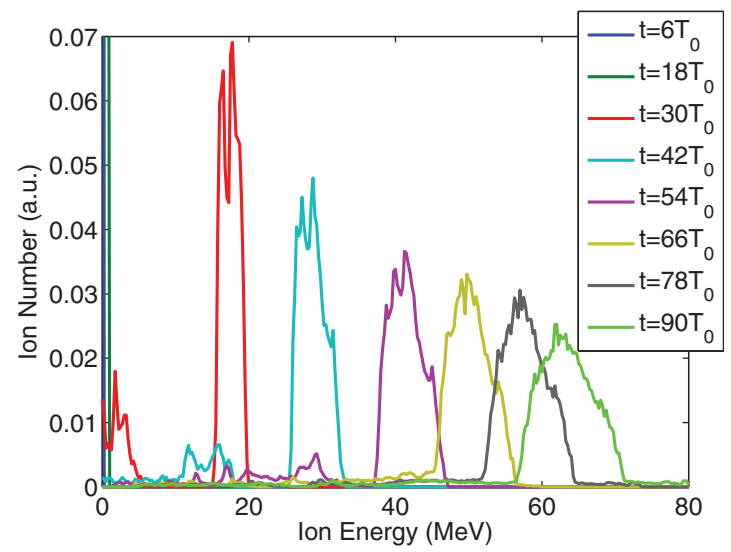

FIG. 7. Evolution of the proton energy spectrum for a target with smaller (located in $-0.5<\mathrm{y}<0.5$ ) $\mathrm{H}$ layer.

the simulation result for a hydrogen layer of lateral size $1 \mu \mathrm{m}$ (centered on the axis), keeping the laser and Al-layer parameters the same as before. One can see that the proton spectrum is indeed improved.

\section{DISCUSSION}

For the given laser parameters, we found that neither the $\mathrm{Al}$ nor the $\mathrm{H}$ layer can be too thick or too thin. If the $\mathrm{Al}$ layer is too thin, the laser can penetrate through the target, so that RPA becomes less effective, and Weibel- or RayleighTaylor-like instabilities can also occur. If the Al layer is too thick, it blocks too many hot electrons, so that fewer TNSA protons can pass through it. As for the $\mathrm{H}$ layer, if it is too thin, there will be too few hot electrons to pull out enough protons. If it is too thick, the hot electrons will pull out too many protons (more than one layer) for the latter to be accelerated effectively over the aluminum layer.

In this scheme, the H-layer density must be much lower than that of the $\mathrm{Al}$ layer. So, we vary the density of the front layer to get more stable experimental conditions. We have also investigated a target with $0.07 \lambda_{0} \mathrm{H}$ and $0.05 \lambda_{0} \mathrm{Al}$ layers

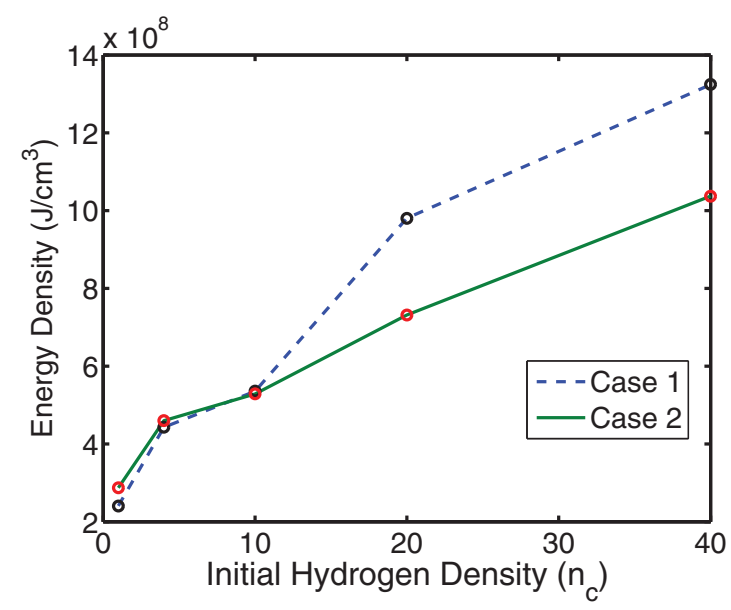

FIG. 8. Energy density at $t=90 T_{0}$ for two targets with different initial $\mathrm{H}$-layer densities and Al-layer densities. Blue dashed curve: Al layer thickness $0.05 \lambda_{0}, \mathrm{H}$ layer thickness $0.07 \lambda_{0}$. Green solid curve: Al layer thickness $0.1 \lambda_{0}, \mathrm{H}$ layer thickness $0.1 \lambda_{0}$. The other parameters are the same as those in Fig. 7. 
and another with $0.1 \lambda_{0} \mathrm{H}$ and $\mathrm{Al}$ layers, for initial $\mathrm{H}$ densities between $4 n_{c}$ and $40 n_{c}$. As Fig. 8 shows, for $\mathrm{H}$ densities between $4 n_{c}$ and $10 n_{c}$, the proton energy density is almost independent of the relative thicknesses of the layers. The first case yields an asymptotic proton density of about $0.028 n_{c}$ at $93 \mathrm{MeV}$ average energy. The second yields a proton density of about $0.046 n_{c}$ at $60 \mathrm{MeV}$ average energy.

In conclusion, using 2D PIC simulations, we have investigated generation of high quality proton bunch from a thin double-layer target irradiated by a high intensity laser pulse. The target consists of a low density $\mathrm{H}$ layer and a higher density Al layer. This configuration prevents the laser from passing through the target and hinders the occurrence of transverse instabilities. The leading part of the TNSA protons can be accelerated through the Al layer into a quasimonoenergetic proton bunch.

\section{ACKNOWLEDGMENTS}

We thank Han $\mathrm{Xu}$ for useful discussions. This work was supported by DOE Grant No. DE-SC-000-1481 and NNSFC Grant No. 10835003.

${ }^{1}$ V. Malka, J. Faure, Y. A. Gauduel, E. Lefebvre, A. Rousse, and K. T. Phuoc, Nature Phys. 4, 447-453 (2008).

${ }^{2}$ K. Krushelnick, Z. Najmudin, and A. E. Dangor, Laser Phys. Lett. 4, 847-862 (2007).

${ }^{3}$ S. P. D. Mangles, B. R. Walton, Z. Najmudin, A. E. Dangor, K. Krushelnick, V. Malka, M. Manclossi, N. Lopes, C. Carias, G. Mendes, and F. Dorchies, Laser Part. Beams 24, 185-190 (2006).

${ }^{4}$ S. Bulanov and V. Khoroshkov, Plasma Phys. Rep. 28, 453 (2002).

${ }^{5}$ A. J. Mackinnon, P. K. Patel, R. P. Town, M. J. Edwards, T. Phillips, S. C. Lerner, D. W. Price, D. Hicks, M. H. Key, S. Hatchett, S. C. Wilks, M. Borghesi, L. Romagnani, S. Kar, T. Toncian, G. Pretzler, O. Willi, M. Koenig, E. Martinolli, S. Lepape, A. Benuzzi-mounaix, P. Audebert, J. C. Gauthier, J. King, R. Snavely, R. R. Freeman, and T. Boehlly, Rev. Sci. Instrum. 75, 3531 (2004).

${ }^{6}$ M. Roth, T. E. Cowan, M. H. Key, S. P. Hatchett, C. Brown, W. Fountain, J. Johnson, D. M. Pennington, R. A. Snavely, S. C. Wilks, K. Yasuike, H. Ruhl, F. Pegoraro, S. V. Bulanov, E. M. Campbell, M. D. Perry, and H. Powell, Phys. Rev. Lett. 86, 436 (2001).

${ }^{7}$ S. C. Wilks, A. B. Langdon, T. E. Cowan, M. Roth, M. Singh, S. Hatchett, M. H. Key, D. Pennington, A. Mackinnon, and R. A. Snavely, Phys. Plasmas 8, 542 (2001).

${ }^{8}$ M. Allen, Y. Sentoku, P. audebert, A. Blazevic, T. Cowan, J. Fuchs, J. C. Gauthier, M. Geissel, M. Hegelich, S. Karsch, E. Morse, P. K. Patel, and M. Roth, Phys. Plasmas 10, 3283 (2003).

${ }^{9}$ P. Mora, Phys. Rev. Lett. 90, 185002 (2003).

${ }^{10}$ J. Fuchs, P. Antici, E. D’Humieres, E. Lefebvre, M. Borghesi, E. Brambrink, C. A. Cecchetti, M. Kaluza, V. Malka, M. Manclossi, S. Meyroneinc, P. Mora, J. Schreiber, T. Toncian, H. Pepin, and P. Audebert, Nature Phys. 2, 48 (2006).

${ }^{11}$ V. I. Veksler, Sov. J. Atom. Energy 2, 525 (1957).

${ }^{12}$ T. Esirkepov, M. Borghesi, S. V. Bulanov, G. Mourou, and T. Tajima, Phys. Rev. Lett. 92, 175003 (2004).
${ }^{13}$ A. P. L. Robinson, M. Zepf, S. Kar, R. G. Evans, and C. Bellei, New J. Phys. 10, 013021 (2008).

${ }^{14}$ O. Klimo, J. Psikal, J. Limpouch, and V. T. Tikhonchuk, Phys. Rev. ST Accel. Beams 11, 031301 (2008).

${ }^{15}$ A. Macchi, S. Veghini, and F. Pegoraro, Phys. Rev. Lett. 103, 085003 (2009).

${ }^{16}$ B. Qiao, M. Zepf, M. Borghesi, and M. Geissler, Phys. Rev. Lett. 102, 145002 (2009).

${ }^{17}$ M. Chen, T. P. Yu, A. Pukhov, and Z. M. Sheng, New J. Phys. 12, 045004 (2010).

${ }^{18}$ S. V. Bulanov, E. Yu. Echkina, T. Z. Esikepov, I. N. Inovenkov, M. Kando, F. Pegoraro, and G. Korn, Phys. Rev. Lett. 104, 135003 (2010).

${ }^{19}$ E. Fourkal, I. Velchev, and C.-M. Ma, Phys. Rev. E 71, 036412 (2005).

${ }^{20}$ L. Yin, B. J. Albright, B. M. Hegelich, K. J. Bowers, K. A. Flippo, T. J. T. Kwan, and J. C. Fernandez, Phys. Plasmas 14, 056706 (2007).

${ }^{21}$ B. M. Hegelich, B. J. Albright, J. Cobble, K. Flippo, S. Letzring, M. Paffett, H. Ruhl, J. Schreiber, R. K. Schulze, and J. C. Fernandez, Nature 439, 441-444 (2006).

${ }^{22}$ H. Schwoerer, S. Pfotenhauer, O. Jackel, K.-U. Amthor, B. Liesfeld, W. Ziegler, R. Sauerbrey, K. W. D. Ledingham, and T. Esirkepov, Nature 439, 445-448 (2006)

${ }^{23}$ Y. W. Tian, W. Yu, P. X. Lu, H. Xu, V. K. Senecha, A. L. Lei, B. F. Shen, and X. Wang, Phys. Plasmas 15, 053105 (2008).

${ }^{24}$ Y. Yin, W. Yu, M. Y. Yu, A. L. Lei, X. Q. Yang, H. Xu, and V. K. Sencha, Phys. Plasmas 15, 093106 (2008).

${ }^{25}$ S. P. Hatchett, C. G. Brown, T. E. Cowan, E. A. Henry, J. S. Johnson, M. H. Key, J. A. Koch, A. B. Langdon, B. F. Lansinski, R. W. Lee, A. J. Machinnon, D. M. Pennington, M. D. Perry, T. W. Phillips, M. Roth, T. C. Sangster, M. S. Singh, R. A. Snavely, M. A. Stoyer, S. C. Wilks, and K. Yasuike, Phys. Plasmas 7, 2076 (2000).

${ }^{26}$ R. A. Snavely, M. H. Key, S. P. Hatchett, T. E. Cowan, M. Roth, T. W. Phillips, M. A. Stoyer, E. A. Henry, T. C. Sangster, M. S. Singh, S. C. Wilks, A. Mackinnon, A. Offenberger, D. M. Pennington, K. Yasuike, A. B. Langdon, B. F. Lasinski, J. Johnson, M. D. Perry, and E. M. Campbell, Phys. Rev. Lett. 85, 2945 (2000).

${ }^{27}$ M. Zepf, E. L. Clark, F. N. Beg, R. J. Clarke, A. E. Dangor, A. Gopal, K. Krushelinck, P. A. Norreys, M. Tatarakis, U. Wangner, and M. S. Wei, Phys. Rev. Lett. 90, 064801 (2003).

${ }^{28}$ I. Spencer, K. W. D. Ledingham, P. Mckenna, T. Mccanny, R. P. Singha, P. S. Foster, D. Neely, A. J. Langley, E. J. Divall, C. J. Hooker, R. J. Clarke, P. A. Norreys, E. L. Clark, K. Krushelnick, and J. R. Davies, Phys. Rev. E 67, 046402 (2003).

${ }^{29}$ G. Doumy, F. Quere, O. Gobert, M. Perdrix, Ph. Martin, P. Audebert, J. C. Gauthier, J.-P. Geindre, and T. Wittmann, Phys. Rev. E 69, 026402 (2004).

${ }^{30}$ S. S. Bulanov, A. Brantov, V. Yu. Bychenkov, V. Chvykov, G. Kalinchenko, T. Matsuoka, P. Rousseau, S. Reed, V. Yanovsky, D. W. Litzenberg, K. Krushelnick, and A. Maksimchuk, Phys. Rev. E 78, 026412 (2008).

${ }^{31}$ Z. M. Zhang, X. T. He, Z. M. Sheng, and M. Y. Yu, Phys. Plasmas 18, 023110 (2011).

${ }^{32}$ H. Xu, W. W. Chang, H. B. Zhuo, L. H. Chang, and Z. W. Yue, Chin. J. Comput. Phys. 19, 305 (2002).

${ }^{33}$ W. Yu, M. Y. Yu, H. Xu, Y. W. Tian, J. Chen, and A. Y. Wong, Laser Part. Beams 25, 631-638 (2007).

${ }^{34}$ S. C. Wilks and W. L. Kruer, IEEE J. Quantum Electron. 33, 22 (1997).

${ }^{35} \mathrm{http}: / /$ www.ph.utexas.edu/ utlasers/tpp.php

${ }^{36}$ J. Wesson, Tokamaks (Clarendon, Oxford, 2004).

${ }^{37}$ F. Pegoraro and S. V. Bulanov, Phys. Rev. Lett. 99, 065002 (2007). 